\title{
Wide field AO correction: the Large Wavefront Sensor Detector of ARGOS
}

\author{
Gilles Orban de Xivry ${ }^{a}$, Sebastian Rabien ${ }^{a}$, Lothar Barl ${ }^{a}$, Simone Esposito ${ }^{b}$, Wolfgang \\ Gaessler $^{c}$, Michael Hart ${ }^{d}$, Matthias Deysenroth ${ }^{a}$, Hans Gemperlein ${ }^{a}$, Lothar Strüder ${ }^{e}$ and \\ Julian Ziegleder ${ }^{a}$ \\ ${ }^{a}$ Max Planck Institut für extraterrestrische Physik, Garching, Germany \\ ${ }^{b}$ Osservatorio Astrofisico di Arcetri, Firenze, Italy \\ ${ }^{c}$ Max Planck Institut für Astronomie, Heidelberg, Germany \\ ${ }^{d}$ Steward Observatory, University of Arizona, Tucson, Arizona, USA \\ ${ }^{e}$ Max Planck Institut Semiconductor Laboratory, Munich, Germany
}

\begin{abstract}
Wide field correction allowing large field to benefit from adaptive optics (AO) is challenging in more than one aspect. We address here the wavefront sensor (WFS) detector side where, in addition to high sensitivity and low noise, the simultaneous detection of multiple laser beacons and the large number of sub-apertures in a ShackHartmann WFS require a detector to have a large imaging area while preserving a very high readout frame rate. The detector considered has a frame area of $264 \times 264$ pixels with a pixel size of 48 microns. By splitting the image into two framestore areas during readout, repetition rates of more than 1000 frames per second can be achieved. The electronic noise contribution is approximately 3 electrons at the operating temperature. We therefore analyze its performances, showing it fulfills the requirements, in a wavefront sensing application: the measurement of centroids in the case of a Shack-Hartmann WFS for the ARGOs AO project.
\end{abstract}

Keywords: Adaptive Optics Wavefront Detector - pnCCD - Centroids Calculation

\section{INTRODUCTION}

By improving the imaging and spectroscopic capabilities, adaptive optics (AO) has proved to be very useful for ground based telescopes, in particular to current 8 to 10 meter ones. While single conjugated AO suffers from angular anisoplanatism, multiple laser guide stars provide the possibility to correct atmospheric turbulence over a wide field of view. The next generation of extremely large telescopes (ELT, GMT, and TMT) will require, by their very large apertures, large pixel arrays in order to correct the wavefront over their entire aperture and control their deformable mirrors. Future extreme adaptive optics (i.e. adaptive optics for optical wavelength) systems will require high wavefront sampling, both spatially and temporally.

In view of these challenges, the wavefront sensor detectors are critical components of AO systems. Consequently, innovative and specific developments (e.g. [1, for a review]) have been made on various detectors to meet the present and future requirements such as very high frame rates, large pixel area, low read noise and dark current, and high quantum efficiency.

Argos (see for a review [2, in this proceeding]) is a ground layer adaptive optics system at the Large Binocular Telescope (LBT). While providing a multiple laser guide star facility (3 per LBT eye), the primary goal of ARGOS is to enhance the imaging and spectroscopic capabilities for both LUCIFER ${ }^{3}$ instruments over a wide field of view and implement a robust sytem with high observing efficiency. In contrast to usual single conjugated adaptive optics, Argos will not reach the diffraction limit of the telescope but it will be capable of enhancing the image quality and the ensquared energy by a factor 2 to 3 for the LUCIFER instruments.

ARGOS will simultaneously detect the backscattered light from these three laser beacons and will have a large

Further author information: (Send correspondence to G. Orban de Xivry)

E-mail: xivry@mpe.mpg.de

Adaptive Optics Systems II, edited by Brent L. Ellerbroek, Michael Hart, Norbert Hubin, Peter L. Wizinowich, Proc. of SPIE Vol. 7736, 77365C - @ 2010 SPIE · CCC code: 0277-786X/10/\$18 - doi: 10.1117/12.857230 
number of sub-apertures. This constraints the WFS detector to have a large imaging area together with a very high readout frame rate while preserving its sensitivity and low noise. To achieve this, the WFS detector of Argos was chosen to be a pnCCD.

Originally designed for X-ray detection, the pnCCD, from Halbleiter Labor (HLL)/pnSensor, was also shown to be an excellent solution for high-speed optical imaging ${ }^{4,5}$ such as in adaptive optics. Indeed, the pnCCD has very high frame rates up to $1 \mathrm{kHz}$ together with a large imaging area (256x248 pixels). It has also excellent sensitivity over a wide wavelength range, low read-out noise and low dark current. Considering the various characteristics and qualities of the pnCCD, it has been chosen as the wavefront sensor detector for ARGOs where a single detector will simultaneously sense the wavefront residual errors from three laser beacons per each eye of the LBT.

This paper is organized as follows. In section 2, we review the ARGOS requirements on the detector. In section 3 , we describe the pnCCD, the camera package of the ARGOS wavefront detector, and the general performance of the pnCCD. Finally, in section 4, we review how the detector will be integrated into the ARGOS system and present the centroiding algorithm scheme together with a first measurement of the accuracy obtained with the pnCCD.

\section{ARGOS DETECTOR REQUIREMENTS}

As already mentioned, ARGOs is a GLAO project, it will have three LGS per LBT eye, will use the ShackHartmann wavefront sensing technique and will operate under various seeing conditions.

Under these considerations, the main requirements and constraints for the ARGOS wavefront detector are as follows:

- Simultaneous detection of $3 \mathrm{SH}$ patterns (to ease the optical design, the overall implementation, and to have a more compact WFS). Each SH pattern will have 15 sub-apertures on the pupil diameter, each one being 8-by-8 pixels large.

- The WFS detector will be fed with monochromatic light at $532 \mathrm{~nm}$.

- The AO update rate will be $\sim 1 \mathrm{kHz}$. Therefore the frame rate of the detector should be of the same order and will be synchronized with the pulses of the ARGOs lasers.

- The number of photons per milliseconds per sub-aperture will be typically $\sim 1800$ and in the worst case $\sim 700$.

- The accepted laser spots FWHM will be between 0.5 to 2.5 arcseconds.

These requirements lead to the necessity of a very good detector with large imaging area, low noise, high quantum efficiency, low dark current, good linearity, defect-free, and good spatial characteristics (PSF).

As we will present, the pnCCD, chosen as the wavefront detector of ARGOs, provides a nice solution to our needs.

\section{CAMERA CHARACTERISTICS}

\section{1 pnCCD design: principle, format and readout}

Originally, the pnCCD was designed for X-ray telescopes by HLL/pnSensor (XMM-Newton ${ }^{6}$, eRosita). The detector concept is based on the principle of sideward depletion of a pnp-structure. By adequate voltages at the front and backside of the CCD, full depletion is achieved resulting in a detector with high quantum efficiency (QE).

Figure 1(a) shows a schematic cross-section of a pnCCD along one transfer channel. The pnCCD is fabricated from high purity n-type silicon. The back side is used as the entrance window, it consists in a homogeneous and very thin $\mathrm{p}+$ implantation and is covered by an anti-reflective coating. On the front side, the transfer registers

Proc. of SPIE Vol. $773677365 \mathrm{C}-2$ 
are formed by $\mathrm{p}+$ implantations. The basic principles is therefore as follow: the strong electric fields in the pnCCD detector separate the electrons and holes before they can recombine. The electrons drift to the potential minimum (inside a lower resistivity layer) and are stored under the transfer registers (which define the pixels). The holes, on their side, move to the negative back side (where they are "absorbed"). After integration, the transfer is performed by a 3-phase set of registers towards the readout anode (see for example [6, for a review of the concept of the pnCCD]). For each channel of the CCD, a JFET is monolithically integrated for on-chip signal amplification which in its turn is connected to the input stage of a multi-channel analog signal processor.

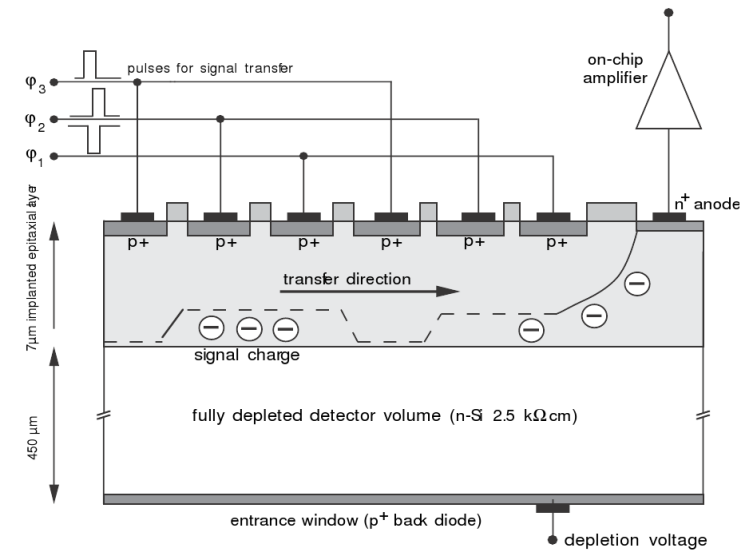

(a) Cross-section of the pnCCD.

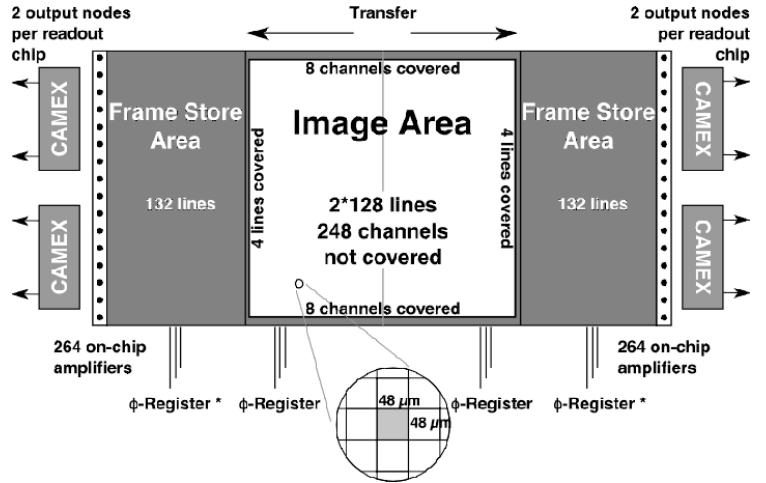

(b) Schematic layout of the pnCCD designed for high speed imaging. The read-out is operated in a splitframe-transfer mode.

Figure 1. pnCCD detector: schematic cross-section and layout.

A schematic illustration of the pnCCD is represented Figure 1(b). The detector format consists in an image area of $248 \times 256$ pixels, $48 \times 48 \mu \mathrm{m}^{2}$ each, forming a $11.9 \times 12.2 \mathrm{~mm}^{2}$ area. To allow high frame rate operations, the pnCCD detector was designed to operate in a split-frame-transfer mode. Therefore, each half image is transferred to its storage region on opposite sites of the detector within $30 \mu \mathrm{s}$. The signal readout is accomplished by four readout ASICs (CMOS Amplifier and MultiplEX or CAMEX ${ }^{7}$ ) having 132 channels each. Eight reference channels (columns) and four reference lines are implemented on each side of the detector to correct for timevariant offsets (to correct for the so called common-mode variations, a line-by-line variations, and to possibly monitor time dependent offset variations). A photography of the pnCCD is given in Figure 2 mounted with four CAMEX readout ASICs on a ceramic carrier board.

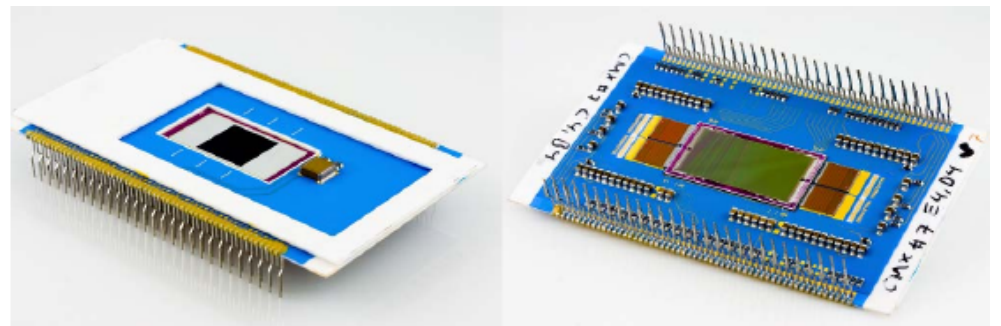

Figure 2. Photography of a $48 \mu \mathrm{m}$ pnCCD, mounted on a ceramic substrate. (Left) Entrance window side, back side of the detector. (Right) Electronics side with the four CAMEX units.

The readout of the pnCCD being column parallel, the frontend of each CAMEX multiplexes the outputs into two outputs nodes, each one being linked to a single external ADC. To allow high-speed applications, a specific read-out scheme is used (see [4, for a description]) which limits the total number of electrons that can be measured by the voltage saturation of internal amplifiers in the CAMEXs. 


\subsection{Camera Package: Electronics and Mechanical Designs}

Mechanical Design. A new detector housing has been designed at HLL/pnSensor. At the time of writing, we just received the first model and we are currently in an evaluation phase (see Figure 3).

The objectives of this design are two folds: to be compact and to allow the use of either nitrogen or vacuum (vacuum-proof) in order to ease the integration to the WFS system and have low maintenance needs. The main characteristics of this new housing are:

- The cooling is provided by two Peltier elements and a water heat exchanger.

- The backside of the camera consists of the electronic boards that allow to drive the pnCCD.

- The back of the housing itself provides the different interfaces points, i.e. the heat exchanger tubes, the electrical interfaces for the pnCCD control, the electrical feedthroughs for Peltier elements and sensors (temperature and pressure), and a valve for vacuum pumping or nitrogen flushing.

- The front side consists in the entrance window of the camera. A flange provides several interface points as well.

- The detector housing interior consists in a multi-layers materials that allows to cool the CCD chip and evacuate the heat generated by the CAMEXs, and provide the necessary radiation and convection shieldings.

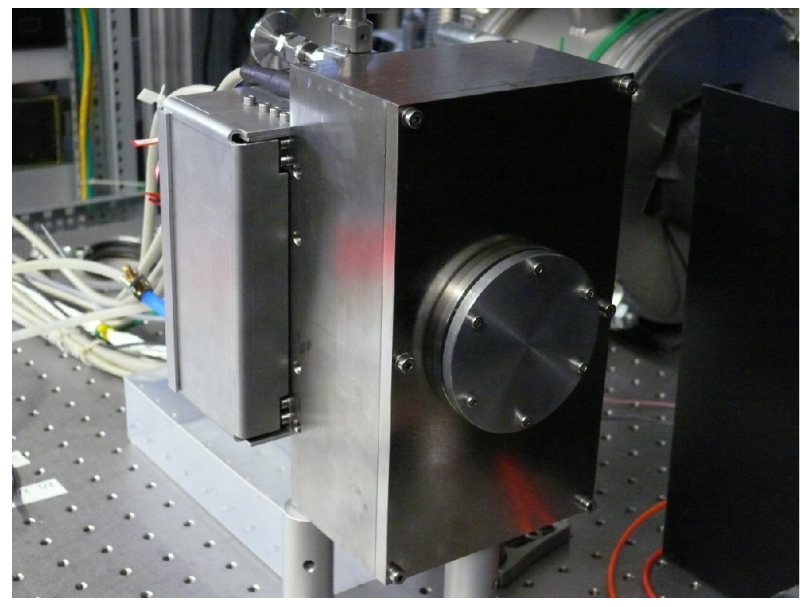

(a) Front side.

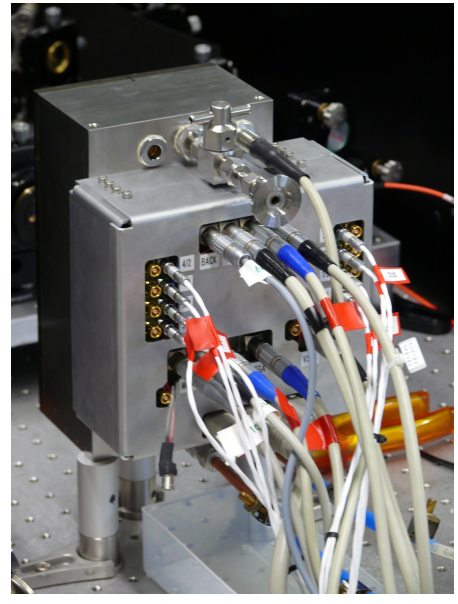

(b) Back side.

Figure 3. The new housing of the camera.

Electrical Design. Two setups have been foreseen for the detector: one for laboratory allowing detector qualification and one for the final system at the telescope. The main differences are in the CCD control and the acquisition unit:

- In the laboratory setup, the CCD control and acquisition are done through a normal computer and use a cPCI interface.

- In the final setup, a compact cPCI CPU will perform the CCD control, the data on their side will go directly from the 2 Data Acquisition Boards (each one having 4 ADCs) to a real time computer (thereafter Basic Computing Unit or BCU) through 2 optical links, allowing the fast data rate needed.

The remaining electronics are common to both setups and consist of the power supplies, the housekeeping elements, and the Data Acquisition Boards. Because the power supplies are relatively large, we are currently designing a more compact power supply at MPE to shrink the readout electronics crate to 6 rack units while preserving performance. The current objective is therefore as shown in Figure 4(a). A 3D model picture of one of the two power supply boards is shown in Figure 4(b). 


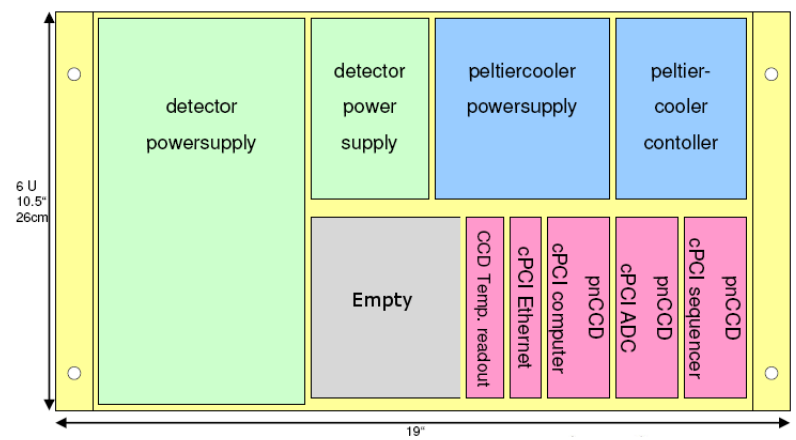

(a) The current layout of the electronics detector racks.

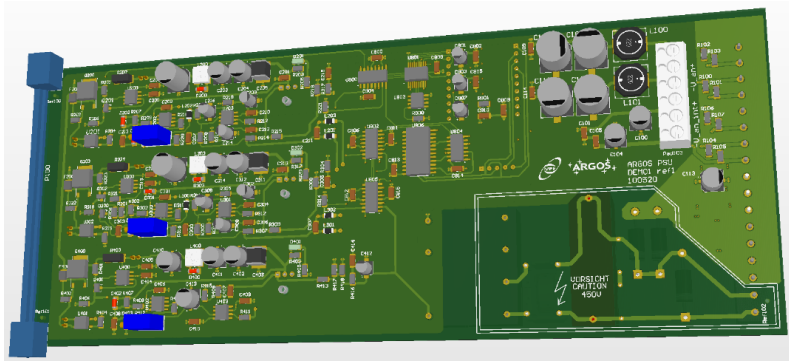

(b) Front side of one of the two power supply boards, currently in design at MPE.

Figure 4. The electronics arrangement and current design of a new power supply at MPE.

\subsection{Detector performance}

The pnCCD allows different read-out modes. For our application, a frame rate of $1 \mathrm{kHz}$ is required, we therefore use the fast read-out mode which limits the useful illumination range and slightly increases the noise ${ }^{4}$ compared to the low read-out mode.

In its fast read-out mode, the detector allows several gain modes that enable one to make the trade off between the useful range of illumination and the read-out noise. For ARGos, the higher gain - which also has lower noise - does not provide the illumination range needed. We therefore select the second gain setting which is 4 times smaller. In the following, we present some key characteristics of the detector in this fast read-out mode and low gain setting. Some results come from previous measurements from HLL/pnSensor, some others have been done independently at MPE. The different results are summarized Table 1.

As the pnCCD was originally developed for X-ray detection, when used with adapted anti-reflective coating (ARC), it allows a high quantum efficiency above $90 \%$. Figure $5(\mathrm{a})$ shows the quantum efficiency (QE) of the pnCCD where the two curves show the QE for extremal values of the layer thicknesses of ARC.

The Point Spread Function (PSF), on its side, gives a measure of the spatial performance of the detector, telling us how accurately the detector determines where the photons arrives. Figure 5(b) was obtained by moving a point-like source over the detector. The charge collected inside the analysis region with respect to its position can be described by an error function, the Figure 5(b) being the derivative with respect to the position of the charge collected. Two substrate voltages and two wavelengths have been studied ${ }^{4,5}$.

The conclusion of these two measurements is simple: the pnCCD has a high quantum efficiency in our wavelength range of interest (optical), and show good PSF characteristics.

Using Photon Transfer Curves, we analyse the useful illumination range and the linearity of the CCD. Since we are working in a fast read-out mode, the amplifiers in the CAMEXs are reset only once every frame ${ }^{4}$, therefore the useful illumination range is limited by voltage saturation within the CAMEX readout ASIC rather than by full depth saturation of the pixels. Taking that into account, the useful illumination range in our gain mode is below $\sim 31250 \mathrm{e}^{-}$per channel or approximately $2000 \mathrm{e}^{-}$per 8 pixels of the same channels, i.e. this gain mode allows a high number of photons per subaperture and saturation will therefore not be a problem. Above this level, the last pixels in a channel start to saturate. The non-linearity in the non-saturated range is below $1 \%$.

The gain and read-out noise histograms for one CAMEX are given Figure 6. The frames used to derive these values have been flat field corrected. If such a correction is not performed, differences between CAMEXs are found to be max $7 \%$. In addition, a flat field map shows small gain variations from channel to channel $(5 \%$ to 10\%). A flat field correction (i.e. gain correction) therefore might be considered depending on the application 


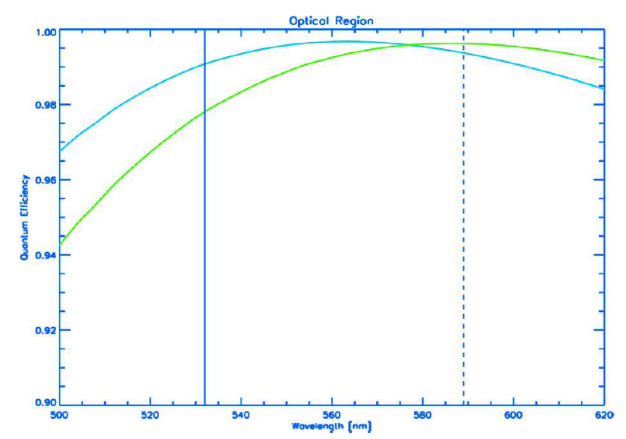

(a) Expected quantum efficiency. The blue and green curves correspond to different layer thicknesses of ARC.
$\%$ of charge in central line of pnCCD versus scan position.

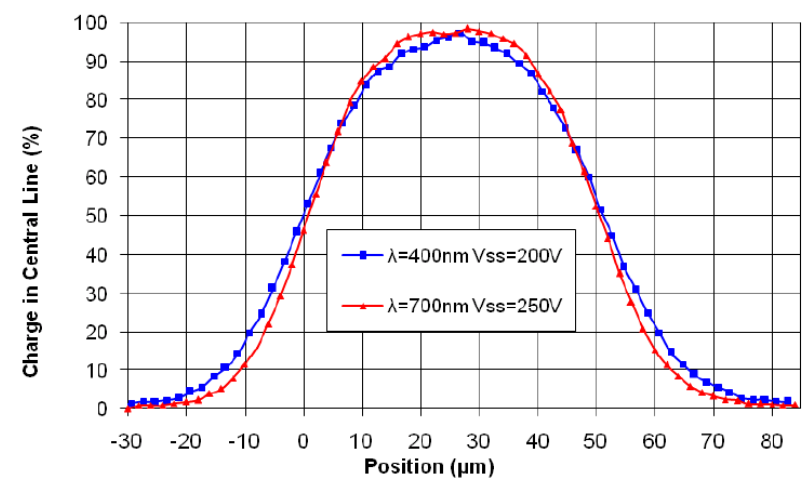

(b) Point Spread Function. Percentage of charge collected in the central line versus scan position of a pnCCD for wavelengths of $400 \mathrm{~nm}$ and $700 \mathrm{~nm}$ and the substrate voltages of $200 \mathrm{~V}$ and $250 \mathrm{~V}^{4,5}$.

Figure 5. Measurements performed by HLL/pnSensor.

and the pnCCD. On ARGos side, we have kept open the possibility to correct these channel to channel variations in case they affect the wavefront reconstruction.

Finally, the dark current is found to increase little with temperature allowing us to operate from -40 to $-15^{\circ} \mathrm{C}$ with an increase of only $\sim 0.1 \mathrm{e}^{-}$in the noise (it allows therefore the use of Peltier elements, less efficient but far more compact than a cryogenic cooling system).

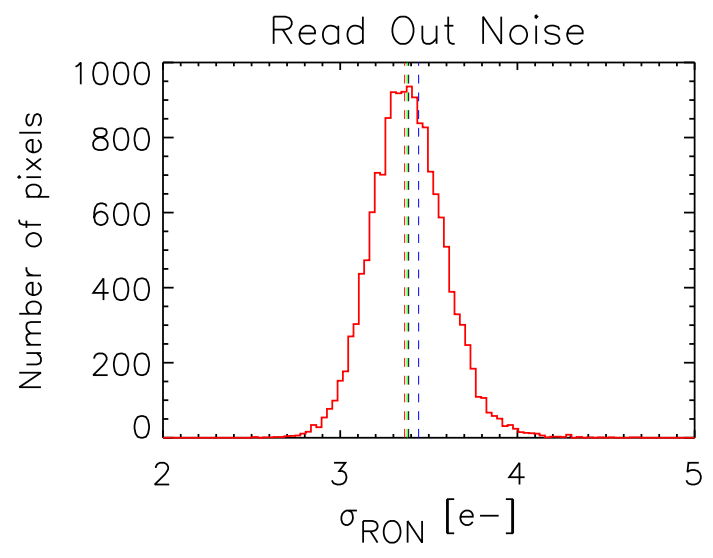

(a) Read-Out Noise at $-35^{\circ} \mathrm{C}$ on the chip

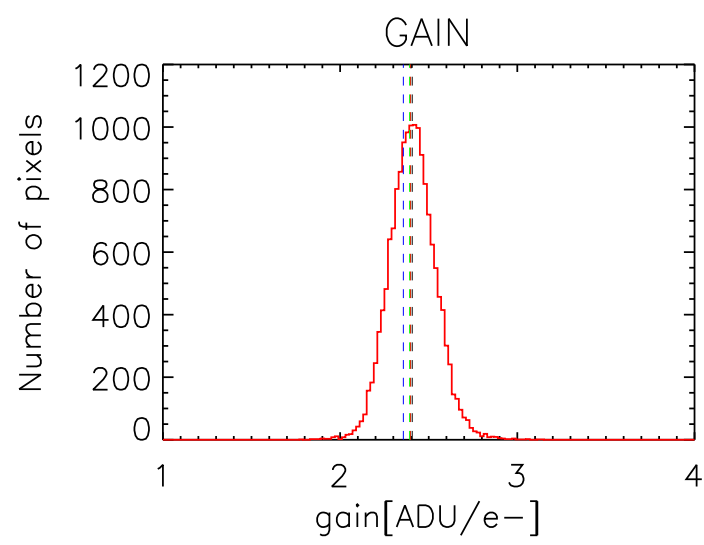

(b) Gain distribution in the low gain mode.

Figure 6. Read-Out Noise and gain distribution for one CAMEX. Medians for the other CAMEX are also represented.

As already mentioned, the detector shows as well a common-mode variation (time varying offset resulting in a line-by-line variation) which can be corrected by using the eight reference channels available per CAMEX. Doing so leads to a good correction and minimizes the residuals.

The different characteristics of the detector are summarized in the table below, Table 1 . 


\begin{tabular}{|c|c|}
\hline Type of CCD & Column-parallel, split frame transfer \\
\hline Total number of pixels & 69696 \\
\hline Pixel size & $48 \mu \mathrm{m} \times 48 \mu \mathrm{m}$ \\
\hline Pixel in image area & $248 \times 256$ pixels \\
\hline Reference pixels & 8 columns and 4 lines per CAMEX \\
\hline Image area & $11.9 \times 12.2 \mathrm{~mm}^{2}$ \\
\hline $\begin{array}{l}\text { Quantum efficiency at } 532 \mathrm{~nm} \\
\text { At }[500 \mathrm{~nm}, 800 \mathrm{~nm}]\end{array}$ & $\begin{array}{l}>98 \% \\
>90 \%\end{array}$ \\
\hline Charge Transfer Efficiency & $>0.99999$ \\
\hline Full well capacitance & $>50000 \mathrm{e}-$ \\
\hline Dark current @- $50^{\circ} \mathrm{C}, 100 \mathrm{fps}$ & $0.196 \mathrm{e}-/ \mathrm{pixel} / \mathrm{s}$ \\
\hline "Hot" pixels & No hot pixels at $20 \mathrm{fps}$ \\
\hline "Dark" pixels & No dark pixels at $900 \mathrm{fps}$ \\
\hline Operating frame rate & $10 \mathrm{~Hz}-1000 \mathrm{~Hz}$ \\
\hline \multicolumn{2}{|c|}{ In fast read out mode, $900 \mathrm{~Hz}$ and $\mathrm{T}=-35^{\circ} \mathrm{C}$} \\
\hline $\begin{array}{l}\text { Difference between left and right CAMEXs: } \\
\text { approx. } 7 \%\end{array}$ & High gain mode \\
\hline Mean read out noise & $2.75 \mathrm{e}^{-}$ \\
\hline Mean conversion factor & $2.4 \mathrm{ADU} / \mathrm{e}^{-}$ \\
\hline Linearity range $(+/-1 \%)$ & $\sim 2000$ to $31250 \mathrm{e}^{-} /$channel $\sim 700$ to $6300 \mathrm{e}^{-} /$channel \\
\hline
\end{tabular}

Table 1. Detector Characteristics summary. QE, CTE, Full Well capacitance, Dark current have been measured by HLL/pnSensor.

\section{OUR APPLICATION: THE WAVEFRONT SENSOR DETECTOR OF ARGOS}

The primary goal of ARGOS, as already mentioned, is to be a robust GLAO system under various seeing conditions enhancing the sensitivity and resolution of LUCIFER instruments, the wide field multi objects spectrographs and NIR imagers ${ }^{3}$ currently in operation at LBT. By shrinking the PSF in various seeing conditions and over a wide field of view, it will widen the scientific capabilities of LUCIFER and enable conducting observations in much shorter time.

Coming from a $12 \mathrm{~km}$ distance, the backscattered light from the laser beacons of ARGOS (see [8, for the laser system description]) will be picked up in front of the instrument rotator structure by a dichroic reflector and directed to the wavefront sensor optical setup.

A detailed description of the wavefront sensor system is given in this proceeding ${ }^{9}$. Figure $7($ a) gives an overview of the arrangement of the WFS. Past the entrance window and a collimating lens, periscope mirrors will bring the light of the three laser beacons closer to the symmetry axis. The second mirror of these periscopes, located at the pupil plane, will be mounted on a fast piezo tip-tilt stage. This fast tip-tilt mirror will stabilize the SH spots within their sub-apertures, compensating any laser beam jitter on the up-link part. Following the periscope mirrors, an optical shutter (based on Pockels Cells) will gate out the desired slice of the atmosphere from the backscattered light. These Pockels Cells will be therefore synchronized with the laser pulses. Finally, a re-focussing lens, a field lens and a common collimator will re-image the pupil onto a lenslet array in front of the pnCCD. Additionally, three cameras (called patrol cameras) will be used to find the laser spot in a field of view of one arcminute in order to help the acquisition process.

The end part being the detector, the pnCCD will simultaneously sense the three SH patterns per LBT eye. The $3 \mathrm{SH}$ disposition provided by the WFS setup is represented in Figure $7(\mathrm{~b})$. With a laser pulse rate of $10 \mathrm{kHz}$ and the Pockels Cells opening after the appropriate delay, the detector will integrate ten backscattered pulses before reading out at a frame rate of $1 \mathrm{kHz}$.

Each SH pattern will provide 175 sub-apertures leading to 350 slopes and will allow ARGos to correct for approx. 150 modes. In addition to this large number of sub-apertures, each of them will be 8-by-8 pixels wide allowing large offsets of the spot positions. Finally, the large pixels $(48 \mu \mathrm{m})$ of the pnCCD together with the 
WFS optical setup will provide a pixel scale of $0.56 \% /$ px which, considering the non-diffracted laser spots we will have, will lead to a good signal to noise ratio while correctly sampling the spots.

These different characteristics will help Argos to be a reliable and robust system with high observing efficiency.

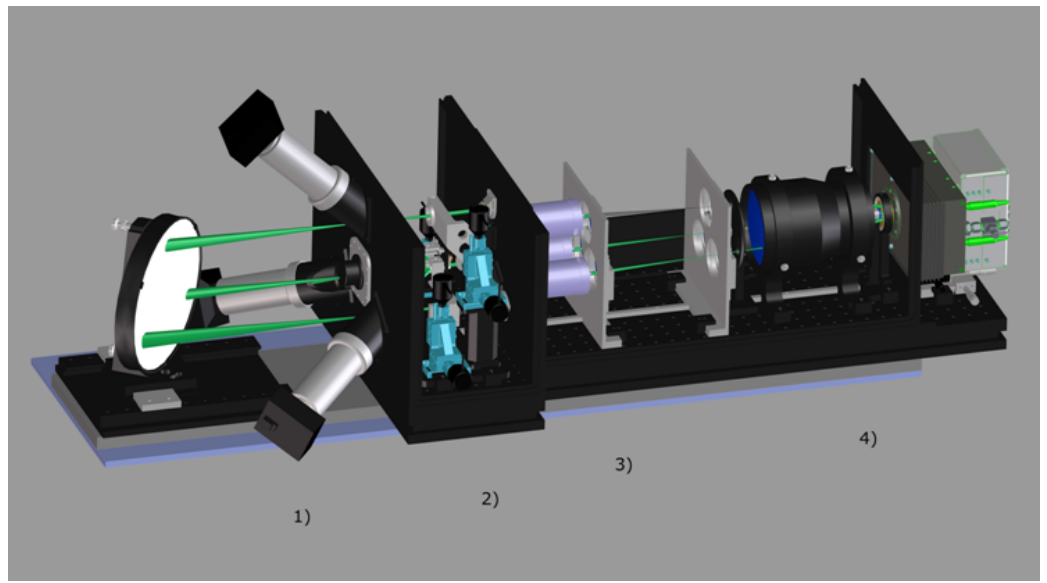

(a) Mechanical arrangement of the WFS setup. ${ }^{9}$

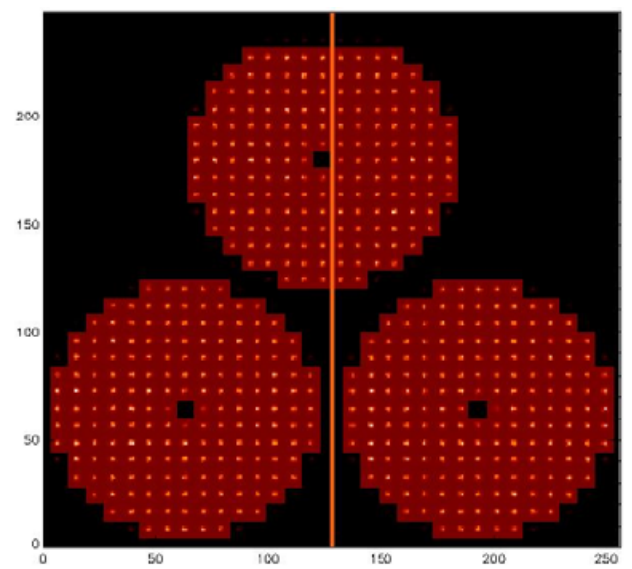

(b) Foreseen disposition of the $3 \mathrm{SH}$ pattern on the detector. The middle line shows the delimitation between the two halves of the read-out (it is not a physical separation).

Figure 7. A steel structure will mount it stiffly to the LBT gallery. Inside the dry air flushed cover, the main elements can be seen: 1) patrol cameras 2) periscope and tip-tilt, 3) Optical gating unit, 4) collimator lenslet and the pnCCD detector.

\subsection{Centroiding accuracy}

The digitized data from the detector unit will be send to a first real time computing unit (BCU). This computer will perform the appropriate corrections of the data (offset, common-mode, and possibly flat field corrections), then it will measure the local slopes (LGS slopes) of the wavefront residual errors and finally send these slopes to the reconstructor for correction with the Adaptive Secondary.

The LGS slopes will also be used to drive the piezo tip-tilt mirrors in the WFS unit to keep the SH centered on the detector.

In order to measure the local slopes, the centroids of each spots need to be computed. The algorithm we have chosen is a generalized center of gravity ( $\mathrm{CoG}$ ) that allow several degree of freedom for optimization while staying relatively simple. It results from different relevant papers ${ }^{10-12}$. The generalized formulation is as followed:

$$
\begin{aligned}
& \forall \text { sub-ap. } a \text { : } \\
& \qquad \begin{aligned}
x_{a} & =\gamma K^{-1} \sum_{i ; I_{i, a}>I_{a ; T}}^{N} x_{i} W_{i, a}\left(I_{i, a}-I_{a ; T}\right)^{n} \\
y_{a} & =\gamma K^{-1} \sum_{i ; I_{i, a}>I_{a ; T}}^{N} y_{i} W_{i, a}\left(I_{i, a}-I_{a ; T}\right)^{n}
\end{aligned}
\end{aligned}
$$

with $\mathrm{K}$, the normalization factor, is defined as :

$$
K=\sum_{i ; I_{i, a}>I_{a ; T}}^{N} W_{i, a}\left(I_{i, a}-I_{a ; T}\right)^{n}
$$

where $i$ is the pixel index within a sub-aperture and $I_{i, a}$ the intensity of the pixel $i$ in the sub-aperture $a$. $W_{i, a}$ is a predefined weighting function sub-aperture dependent. If this weighting function is used, a coefficient $\gamma$ is 
needed to ensure linear response between estimated and real position. The factor $n$ is foreseen to be either 1 or 1.5 (so to weight the pixels by their respective signal to noise ratios ${ }^{12}$ ). The threshold $I_{a ; T}$ could be either a constant value for every sub-apertures, or determined dynamically as to be proportional to the maximum pixel value within the sub-aperture considered ${ }^{10}: I_{a ; T}=\alpha I_{a ; \max }$.

Using an optical setup in our laboratory to create a Shack-Hartmann pattern Figure 8(b), we have tested the centroiding accuracy obtained with our detector and compared it to simulation. We used a simple version of the above algorithm, i.e. a simple threshold at $3 \sigma_{R O N}$ with $W=1, \gamma=1$, and $n=1$. We have simulated similar spots adding photon noise and read-out noise with a random position within one pixel, and we applied the same algorithm. The result is given Figure 8(a).

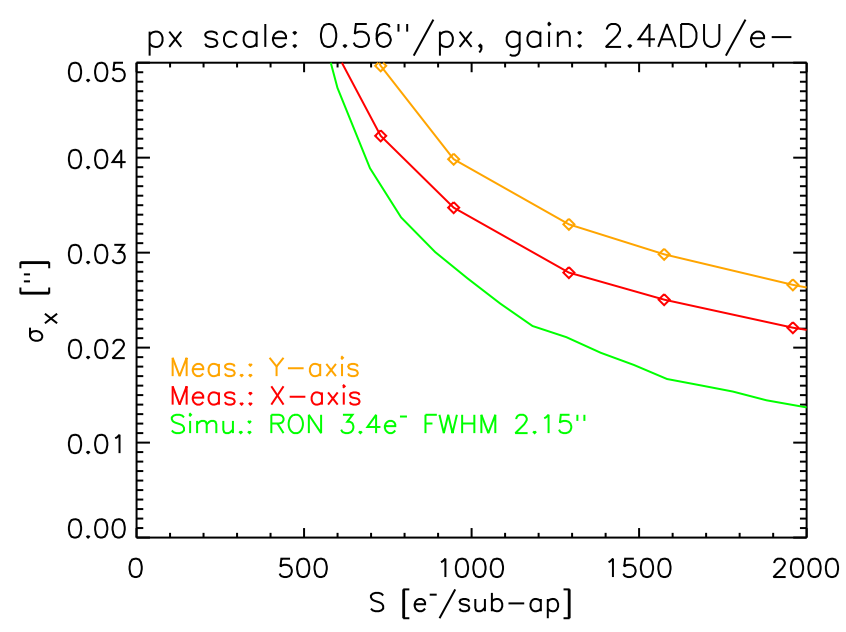

(a) Centroiding accuracy measurement for spots with a FWHM 3.8 pixels, i.e. $2.15 "$.

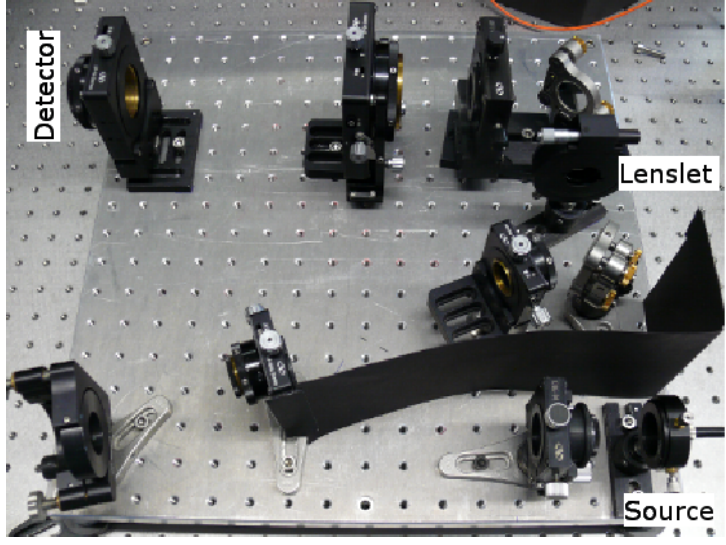

(b) Current laboratory setup that produce $1 \mathrm{SH}$ pattern of 15 by 15 spots.

Figure 8. Test of the centroiding accuracy.

The following remarks can be made about the result:

- The difference between the $x$ (rows) and $y$ (columns/channels, read-out direction) axes can be attributed mainly to charge smearing in the y direction and as well possibly to common-mode residuals. Indeed, in our setup, light is shining continuously on the detector. When the detector is column-parallel read-out, charges are detected at wrong location causing this smearing in the y (column) direction.

- The difference between our simulation and measurement ( $\mathrm{x}$ axis) is attributed to spot to spot contamination and background light that were not considered in the simulation.

Taking these two effects into account, we conclude that the detector meets the requirements of centroiding accuracy. Together with its large imaging area, very high frame rate, high sensibility and low noise, this detector is perfectly suited for the ARGOS AO system.

\subsection{Future Tests and Integration}

The next foreseen steps are the integration of the detector with the WFS optics on one hand, and the integration with the BCU on the other.

The assembly of the camera with the WFS setup will lead to several further tests, such as the verification and adjustment of possible flexure and misalignments between the two parts, the synchronization of the Pockels Cells with the detector read-out, further centroiding tests, etc. 
The integration with the BCU will be composed of the verification, among others, of the data integrity, the communication connections, the several correction and computational steps, the latencies and the control of the fast tip-tilt mirrors.

These future steps will take place in Europe within the following year.

\section{REFERENCES}

[1] Downing, M., Finger, G., Baade, D., Hubin, N., Iwert, O., and Kolb, J., "Detectors for AO wavefront sensing," Adaptive Optics Systems 7015(1), 70151R, SPIE (2008).

[2] Rabien, S., Ageorges, N., Beckmann, U., Blümchen, T., Bonaglia, M., Borelli, J. L., Brynnel, J. G., Busoni, L., Davies, R. I., Deysenroth, M., Durney, O., Esposito, S., Gaessler, W., Gasho, V., H. Gemperlein, R. G., Green, R. F., Hart, M., Kanneganti, S., Masciadri, E., Noenickx, J., Diethard, P., Rademacher, M., Rix, H., Schwab, C., Storm, J., Weigelt, G. P., Orban de Xivry, G., and Ziegleder, J., "ARGOS - the laser guide star system for the LBT," Adaptive Optics Systems , 7736-13, SPIE (2010).

[3] Mandel, H., Seifert, W., Hofmann, R., Jutte, M., Lenzen, R., Ageorges, N., Bomans, D., Buschkamp, P., Dettmar, R.-J., Feiz, C., Gemperlein, H., Germeroth, A., Geuer, L., Heidt, J., Knierim, V., Laun, W., Lehmitz, M., Mall, U., Muller, P., Naranjo, V., Polsterer, K., Quirrenbach, A., Schaffner, L., Schwind, F., Weiser, P., and Weisz, H., "LUCIFER status report: summer 2008," Ground-based and Airborne Instrumentation for Astronomy II 7014(1), 70143S, SPIE (2008).

[4] Hartmann, R., Deires, S., Downing, M., Gorke, H., Herrmann, S., Ihle, S., Kanbach, G., Papamastorakis, J., Soltau, H., Stefanescu, A., and Strüder, L., "Results of a pnCCD detector system for high-speed optical imaging," Adaptive Optics Systems 7015(1), 70155C, SPIE (2008).

[5] Ihle, S., Hartmann, R., Downing, M., Struder, L., Deiries, S., Gorke, H., Hermann, S., Kanbach, G., Papamasturakis, J., Soltau, H., and Stefanescu, A., "Optical test results of fast pnCCDs," in [Nuclear Science Symposium Conference Record, 2008. NSS '08. IEEE], 3351-3356 (19-25 2008).

[6] Strüder, L., Briel, U., Dennerl, K., Hartmann, R., Kendziorra, E., Meidinger, N., Pfeffermann, E., Reppin, C., Aschenbach, B., Bornemann, W., Bräuninger, H., Burkert, W., Elender, M., Freyberg, M., Haberl, F., Hartner, G., Heuschmann, F., Hippmann, H., Kastelic, E., Kemmer, S., Kettenring, G., Kink, W., Krause, N., Müller, S., Oppitz, A., Pietsch, W., Popp, M., Predehl, P., Read, A., Stephan, K. H., Stötter, D., Trümper, J., Holl, P., Kemmer, J., Soltau, H., Stötter, R., Weber, U., Weichert, U., von Zanthier, C., Carathanassis, D., Lutz, G., Richter, R. H., Solc, P., Böttcher, H., Kuster, M., Staubert, R., Abbey, A., Holland, A., Turner, M., Balasini, M., Bignami, G. F., La Palombara, N., Villa, G., Buttler, W., Gianini, F., Lainé, R., Lumb, D., and Dhez, P., "The european photon imaging camera on XMM-newton: The pn-CCD camera," A\&A 365, L18-L26 (Jan. 2001).

[7] Herrmann, S., Buttler, W., Hartmann, R., Meidinger, N., Porro, M., and Strueder, L., "CAMEX readout ASICs for pnCCDs," in [Nuclear Science Symposium Conference Record, 2008. NSS '08. IEEE], 2952 -2957 (19-25 2008).

[8] Kanneganti, S., Rabien, S., Deysenroth, M., Ziegleder, J., Gemperlein, H., and Haug., M., "ARGOS: a laser constellation for adaptive optics at the LBT," Adaptive Optics Systems, 7736-161, SPIE (2010).

[9] Busoni, L., Bonaglia, M., Esposito, S., Carbonaro, L., and Rabien, S., "Final design of the wavefront sensor unit for ARGOS, the LBT's LGS facility," Adaptive Optics Systems , 7736-208, SPIE (2010).

[10] Lardière, O., Conan, R., Bradley, C., Jackson, K., and Hampton, P., "Radial thresholding to mitigate laser guide star aberrations on centre-of-gravity-based Shack-Hartmann wavefront sensors," MNRAS 398, 1461-1467 (Sept. 2009).

[11] Thomas, S., Fusco, T., Tokovinin, A., Nicolle, M., Michau, V., and Rousset, G., "Comparison of centroid computation algorithms in a Shack-Hartmann sensor," MNRAS 371, 323-336 (Sept. 2006).

[12] Kasper, M. E., Looze, D. P., Hippler, S., Davies, R., and Glindemann, A., "Increasing the sensitivity of a Shack-Hartmann Sensor," Proc. Canterbury Conference on Wavefront Sensing and its Application (1999). 